\title{
Study on Development of Translation Ability and Its Constituent Factors
}

\author{
Yanwei Yang \\ Mingde College, Northwestern Polytechnical University, Xi’an, Shaanxi, 710124
}

Keywords: Translation Ability, Constituent Factors, Development Factors

\begin{abstract}
The development of translation ability is the central issue of translation teaching research, which determines the setting, implementation and evaluation of translation courses. The core issues of translation competence research are: What are the sub-abilities of translation ability? Which stages can translators develop into? And how to develop translator's translation ability? Based on the four pairs of contradictions and the study of Chesterman's five-stage translation competence development, six constituent factors of translation ability was discussed in a comprehensive model of modern translation ability. According to the three stages of Chinese translator's translation ability, teachers in China should stress the core ability, the development of one's language ability, and other abilities in translation in order to fully develop translator's translation ability and make them become advanced translators.
\end{abstract}

\section{Introduction}

With the growing demand of social talents for foreign languages and qualified translators of different languages, the research on translation competence has become a hot spot in the field of translation studies. Since the core task of translation teaching is to improve students' translation ability, the study of the constituent factors and development of translation ability is of great importance Interpreting the components of translation ability is not only instructive in translation teaching and translation testing, but also instructive in translation course setting, translation teaching evaluation, and training of translators. ${ }^{[1]}$ For nearly 20 years, researchers have defined translation ability from different perspectives, but till now scholars haven't got a unified understanding of translation ability. ${ }^{[2]}$ This study intends to analyze the components of translation competence by theoretical research, aiming to establish a complete and systematic structure of translation competence to help teachers to improve students' translation ability, to find ways and means to improve students' translation ability and translation teaching efficiency and to help translation researchers carry out further research on the development of translation ability. ${ }^{[3]}$

\section{The Constituent Factors of Translation Ability}

Translating ability is a psychological entity manifested in external behavior. It has different characteristics and manifestations on different sides and stages, which determines the complexity, dynamism, conformity and long-term development of translating ability. ${ }^{[2]}$ In the process of exploring a comprehensive translation ability model, we need to deal with the following four pairs of contradictions: contradictions between external norms and internal transformations, contradiction between behavior and psychology, the conflict between result and process and conflicts between intellectual and non-intellectual factors. ${ }^{[4]}$

Based on the studies of four pairs of contradictions in translation, the intellectual and non-intellectual factors, external factors and internal factors, behavior and psychology, process and result, the comprehensive model of modern translation ability, 6 constituent factors of translator's translation ability, will be proposed in the following paragraphs.

(1) Language competence, including the basic knowledge of two languages, like vocabulary and sentence, etc, language comparison and contrast ability, discourse comprehension and analysis ability, and the ability of aesthetic reproduction of the original texts, and translation realization ability; ${ }^{[4]}$ 
(2) Cultural competence, including cultural cognitive ability, cultural comparative ability, cultural acceptance and tolerance ability and cultural coordination ability;

(3) Strategic competence, including the analysis of translation situations and tasks, the development of translation plans, the selection and application of translation process strategies, and the monitoring and evaluation of translation process.

(4) Tool capabilities, including the ability to use bilingual dictionaries, paired dictionaries, parallel texts, translation corpus, and online resources in the exchange of information with expertise in relevant realm; ${ }^{[2]}$

(5) Thinking ability, including the image of thinking, logical thinking, creative thinking, divergent thinking and other thinking capabilities;

(6) Co-ordination ability, including the coordination of translator's personality and other translators' personalities, the formation of teamwork spirit, the coordination of translator's intellectual and non-intellectual factors and so on. ${ }^{[4]}$

Among these six constituent factors, language competence is the core competence and the ultimate goal of translator training because in comparison to other abilities, which can be improved or acquired through training in a relatively short period of time, it requires long-term and systematic training and directly determines the quality of the translated versions or the final product of translation. Cultural competence influences the readability and acceptance of translation; strategic competence affects the adaption of translation in the target language environment; thinking ability impacts the translator's translation style; tool capabilities are of critical importance in the improvement of translator's translation speed and terminological consistency; and co-ordination ability helps translators from different countries finish the same translation project under big data and cloud data.

\section{Chesterman's Five-stage Translation Competence Development}

Chesterman (2000) proposed five stages in the development of translation competence: 1) Beginner Phase. In this stage, learners consciously learn how to process simple information, and such learning process generally does not consider context very much, but cares more about objective knowledge and rules of conduct. 2) Advanced Learner Phase. Translating teachers and translators themselves begin to consider contextual factors in the process of translation training and practice. 3) Capability Phase. With the increase of language knowledge and translation experience, learners can conduct overall analysis the text or discourse and planning the translation process and strategies according to specific situations. 4) Proficiency Phase. In this stage, translators will find that personal experience and intuition are increasingly important, and at the same time they will use both rational analysis and sensory intuition in their translation practice. 5) Expert Phase. Translators' perceptual intuition dominates in this stage, although the rational monitoring has not disappeared and moved into the background ${ }^{[4]}$. Translators of this stage will have competence of natural conversion between the two languages. As a result, learners complete the transition from conscious to automated decision-making stage in translation.

\section{The Three Levels of Chinese Translators' Translation Ability}

Chinese students begin learning English when they are in Grade Three in primary school, some even in kindergarten, but they still fell the difficulty in communication or translation when they are in colleges or universities. That is because they still don't have enough language accumulation and lack authentic practice, many of these students are not advanced language learners and their language ability needs to be elevated and their language knowledge needs to be activated. Translation ability is the crucial competence that language learners should have to achieve the ultimate goal of language learning--communicating and understanding among nations. According to China's current conditions, Chinese students' translation ability development can be divided into three levels: primary level, intermediate level and advanced level ${ }^{[5]}$. Students of primary level refers to those who are in the stage of language accumulation, words and grammar being the 
focuses of this stage. Students first learning English to sophomores in universities who get CET 4-6 certificates or TEM 4 certificate belong to this level. Students of intermediate level refers to those students who are have the ability of flexible language use, have evaluation ability of language use, and can provide independent and proper solutions to solve language problems in translation. Students from juniors in universities or graduates belong to this stage if they can meet the above requirements. Translators with advanced translation ability should be those ones who have great language ability, translation strategies, cultural knowledge, flexible use of translation tools, strong think ability and excellent morality, and authentic translation experience of more than 3 years, either the translation experience in international companies or in language service organizations, in which the two language are frequently used in their working and the translation practice of more than 300, 000 words is required. Thus translators of this level are professional and proficient ones who have natural conversion of two languages and cultures with the proper application of translation methods and translation tools to conveying the message in original discourses. Knowing the stage of Chinese students' translation ability development will help translation course teachers and students themselves realize what they should do in order to enhance translation ability.

\section{Their Significance to English Teaching and Translation Course}

From the six constituent factors of translation ability, we found that language competence is the core competence and the ultimate goal of translator training. From Chesterman's five-stage translation competence development, we learned some factors like language, ability, translation skills, translation strategies, thinking, and analyzing are very important in translator's translation ability development and in producing excellent translators. As Chinese to English translation is to make Chinese college students translate Chinese texts into non-native language, English, or English translation to Chinese translation is to require them to translate the original text into readable and natural Chinese based on their correct understanding, the bilingual ability of undergraduates and masters is of critical importance in their translation ability development and to some extent needs to be improved. Therefore, the development of their ability to translate Chinese characters into English or English into Chinese characters is very closely related to their language ability: language level (vocabulary, sentences, paragraphs and discourses), grammatical level, ect. From the three stages of Chinese translators' translation ability development, we can still find out that language ability is the base of the development of other abilities. Therefore, English teaching or translation teaching, no matter for non-English majors or for English majors, ought to place stress on acquirement of language knowledge, cultivate students' awareness of the language difference between the two languages, increase students' input and output of two languages, and enrich students' background knowledge so as to pave the way for students' translation ability development to the other two stages. But how to help students acquire language and culture knowledge, and other translation abilities? What can universities and college do?

The choice of teaching materials, the setting of courses, and the teaching model will have great influence on student's translation ability and its development. Universities and colleges can choose the latest book about translation theory and practice with classic translation theories and translation cases and require teacher to add some translation materials on current affairs to make students keep pace with the development of society. They can also set courses on the contrastive study of Chinese and English, comparison and translation between English and Chinese, Chinese culture (Chinese-English), English society and culture, and other relevant courses to improve students' culture awareness, and set courses on CAT to help students to learn to use translation tools. On the choice of teaching model and translation course evaluation model, teachers can adopt the flipped class teaching model and organize more in-class activities like presentation, discussion and group work to help students master the key points in translation teaching, and apply formative assessment model to find students' problems in translation study and practice to help them develop their translation ability 


\section{Conclusions}

Translator's translation ability is composed of six major factors: language competence, cultural competence, strategic competence, tool capabilities, thinking ability and co-ordination ability, among which language competence is the base. Without strong language competence, students' translation ability will stay in the primary level. In order to improve student's translation ability and make them advanced translators, teachers should also cultivate their culture awareness and tolerance, their logical and creative thinking ability, translation tool ability and morality, etc. The research in the development of translator's translation ability should be continued along with the development of modern technology and the development of college students and translators.

\section{Acknowledgements}

Scientific Research Program Funded by Shaanxi Provincial Education Department (Program No.17JK1137)

\section{References}

[1] Yang Zhihong, Wang Kefei. Translation Ability and Its Research [J]. Foreign Language Education. 2010 (06):52 61.

[2] Tong Yahui. PACTE Translation Ability Model [J]. Journal of PLA University of Foreign Languages, 2010 (05) :75 76.

[3] Qian Chunhua. The Components of Translation Ability and the Driving Relations among Them [J]. Foreign Language World, 2012(3):59-65.

[4] Wang Shuhuai, Wang Ruowe. The Constituent Factors and Developmental Stages of Translation Ability [J]. Foreign Language Research. 200895):80-88.

[5] Chesterman, Andrew. Teaching Strategies for Emancipatory Translation [A]. In Christina Schäffner and Beverly Adab (eds.). Developing Translation Competence [C]. Amsterdam: John Benjamins, 2000:77-89.

[6] Wang Xiangling. Study on Translation Ability in the West: Retrospect and Prospect [J]. Journal of Hunan University (Social Science), 2008 (02) :81 83. 